\title{
What is the difference in morphologic features of the lumbar vertebrae between Caucasian and Taiwanese subjects? A CT-based study: implications of pedicle screw placement via Roy-Camille or Weinstein method
}

\author{
Hsi-Hsien Lin ${ }^{1,2}$, Jung-Pan Wang ${ }^{1,2}$, Cheng-Li Lin ${ }^{3}$, Yu-Cheng Yao ${ }^{1,2}$, Shih-Tien Wang ${ }^{1,2}$, Ming-Chau Chang ${ }^{1,2}$ and \\ Po-Hsin $\mathrm{Chou}^{1,2^{*}}$
}

\begin{abstract}
Background: Safe placement of pedicle screws without jeopardizing neurovascular structures medially and anteriorly is important during spine surgery. Inferior breach of pedicle is also dangerous due to low margin of error. Lumbar morphology and identical pedicle orientation at L1 to $L 5$ shown on CT scan of young Taiwanese patients (90 patients) were analyzed and compared with findings reported for Caucasian subjects.

Methods: Previously reported techniques were employed to quantitatively elucidate the parameters regarding lumbar morphology and identical pedicle orientation at each vertebra. The parameters for pedicle angle (PA), pedicle diameter $(P D)$, pedicle axis distance (PAD), midline axis distance (MAD), transverse pedicle axis distance (TPAD) and transverse intertangential angle (TITA) were measured.

Results: Taiwanese subjects had different PA, PD, PAD, MAD at L1 to L5 and TITA at L3 to L5 compared with Caucasian subjects. L5 had the most convergent pedicle axis, the widest PD and the shortest antero-posterior axis morphology.

Conclusions: This study provides detailed information for identifying pedicle orientation during pedicle screw placement and elucidate racial differences in lumbar morphology and pedicle orientation between Taiwanese and Caucasian populations.
\end{abstract}

Keywords: Pedicle screws, Racial difference, Anatomic study, Roy-Camille method, Weinstein method

\section{Background}

Posterior approaches with transpedicular pedicle screw placement for lumbar spines have been proved to be practical, safe, and effective in the treatment of degenerative disease, trauma, scoliosis, infection and tumor

\footnotetext{
* Correspondence: choupohsin@gmail.com

'Department of Orthopedics \& Traumatology, Taipei Veterans General Hospital, No. 201, Sec. 2, Shipai Rd., Beitou District, Taipei City 11217, Taiwan, Republic of China

${ }^{2}$ School of Medicine, National Yang-Ming University, No. 201, Sec. 2, Shipai Rd., Beitou District, Taipei City 11217, Taiwan, Republic of China

Full list of author information is available at the end of the article
}

[1]. Iatrogenic complications due to mal-positioning of pedicle screws include nerve root injury, vascular injury, and hollow organ injury [2].

Two common methods clinically used for pedicle screw placement were Roy-Camille [3] and Weinstein [4] methods, which were advocated using different anatomic landmarks (Fig. 1). The Roy-Camille method [3] was advocated at the landmark of medial of the accessory process and $1 \mathrm{~mm}$ below the facet joint. The Weinstein method [4] was advocated at the lateral and inferior corner of the superior articular face. The Weinstein method for pedicle screw fixation is commonly used in 


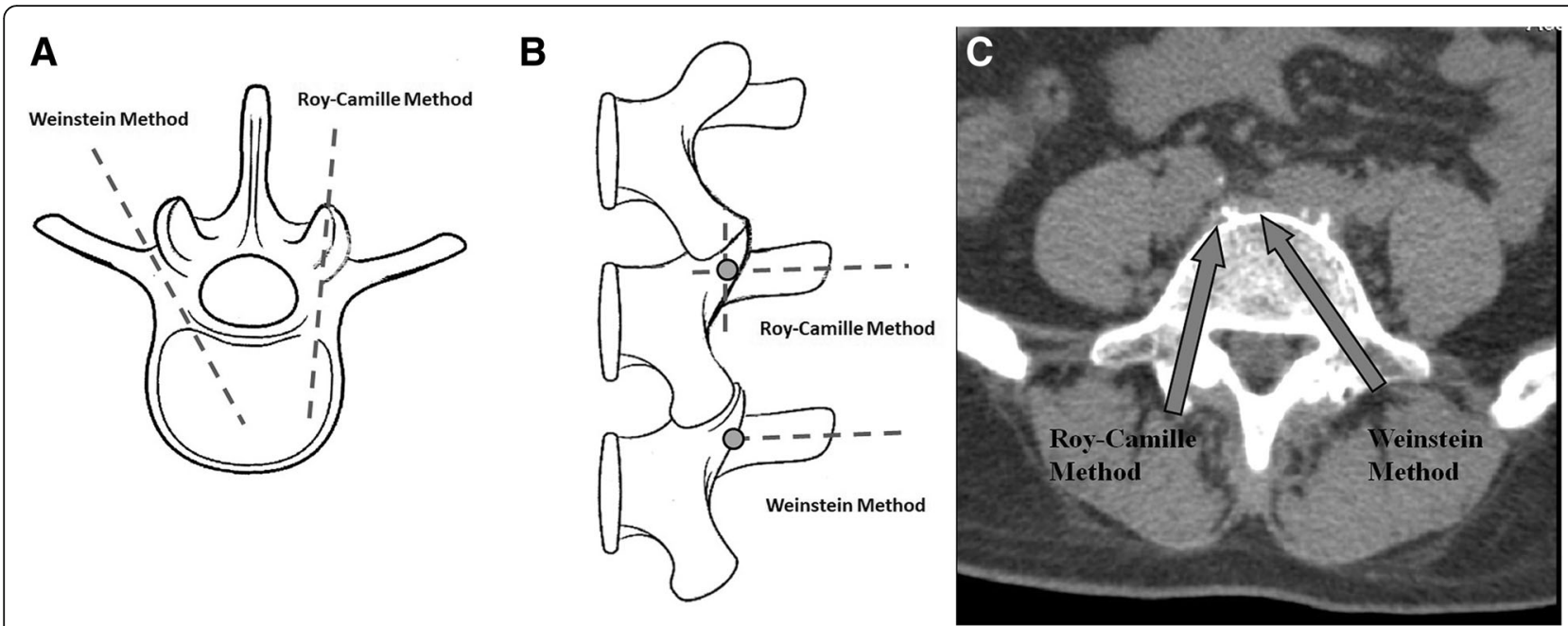

Fig. $\mathbf{1} \mathbf{a}, \mathbf{b}$ and $\mathbf{c}$. Illustration of entry points of pedicle screws via Roy-Camille or Weinstein method. a. Axial view of both methods. $\mathbf{b}$. Posterior view of both methods. c. Roy-Camille or Weinstein method in axial view of CT scan. The gray arrow indicates the trajectory of pedicle screw placement in lumbar spine

the Wiltse approach with paraspinal muscle-sparing [5]. Therefore, it is important for spine surgeons to be familiar with the detailed anatomy of the pedicle orientation in relation to the morphology of each vertebra so as to avoid iatrogenic complications during pedicle screw placement.

Morphometric studies have been performed using direct measurements on cadavers or radiologic measurements in CT scans among different Caucasian [6-8], Indian $[9,10]$, Korean [11] and Chinese [12] populations. Anatomic differences and variations among Caucasian and Taiwanese populations have also been reported [13].

This study analyzed the lumbar morphology and pedicle orientation at L1 to L5 in Taiwanese population. First, pedicle orientation related to the identical lumbar morphology was defined to facilitate the anatomy during pedicle screw placement with either Roy-Camille or Weinstein method. Second, anatomic differences between Caucasian and Taiwanese populations regarding lumbar morphology and pedicle orientation were explored.

\section{Methods}

The research protocol was approved by the institutional review board of Taipei Veterans General Hospital (201710-008 BC). Young patients, aged 20-50 years, who were arranged for CT scan examination due to persisted back pain and sciatica were studied. In other words, the study group was not normal distribution of the Taiwanese population. Computed tomography scans (Toshiba, Aquilion, Tokyo, Japan) of the lumbar spines with 3-mm cut plane were performed and reviewed using a computer software for measurement (Smart viewer 3.2; Taiwan Electronic Data Processing Cooperation, Taipei, Taiwan). Moreover, the measurement plane was defined at the mid-pedicle isthmus in the axial plane, which was determined at the sagittal view.

The inclusion criteria for patients with each lumbar vertebra to be measured were as follows: (1) no previous surgery, (2) no vertebral pathology such as infection, tumor and fracture, (3) no severe degeneration, (4) symmetric pedicle distribution on the axial planes measured, and (5) the cut plane parallel to the inferior endplate. A total of 90 patients met the inclusion criteria for further measurements from January 2014 to September 2014. This number was chosen arbitrarily without a prior power analysis.

There were 52 males and 38 females with mean age at 34.5 years (range, 22 to 46 years). To avoid bias, all radiologic measurements were performed on the radiographs by the same investigator (H.H.L.) to ensure consistent results [14]. So we did not check the inter- or intra-observer bias before all measurements. Three measurements were obtained with their arithmetic mean calculated as data for analysis.

Before measuring each vertebra at different levels, a tangential line was drawn along the bilateral transverse process and another perpendicular midline axis (AP axis) which bisected the vertebral body. The measurement plane was defined at the mid-pedicle isthmus in the axial plane, which was determined at the sagittal view.

The pedicle longitudinal axis was marked through the mid-pedicle level, which was defined as pedicle diameter (PD), as adopted by Olsewski et al. [6] (Fig. 2) The distance from the lamina to the anterior cortex along the AP midline axis was defined as the midline axis distance (MAD), as reported by Zindrick et al. [15] (Fig. 2). The 


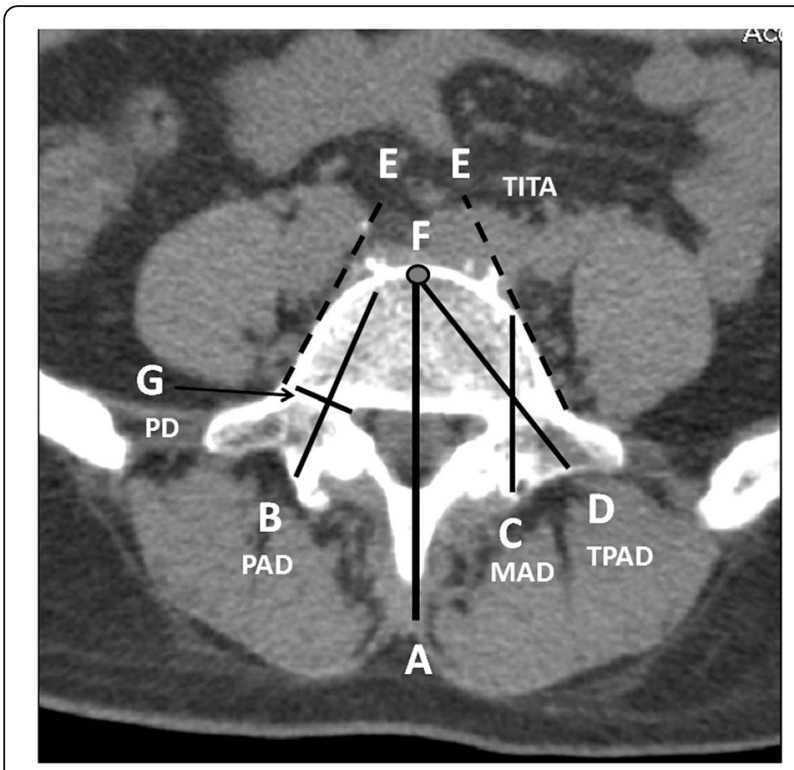

Fig. 2 a to $\mathbf{g}$. Illustration of various radiologic parameters for measurement. a. Antero-posterior (AP) midline axis. b. Distance to anterior cortex along pedicle axis (pedicle axis distance) (PAD). c. Distance to anterior cortex along AP midline axis (midline axis distance) (MAD).d. Distance from interface between transverse process and pedicle to anterior cortex of vertebra along pedicle axis (transverse pedicle axis distance) (TPAD). e. Lateral tangential lines (dotted lines E) along lateral borders of each vertebra. Transverse intertangential angle (TITA) was defined as the angle between two dotted lines $E$ at each side of the vertebra. $\mathbf{f}$. Point $F$ was defined as the anterior border of the vertebra through midline axis and also taken as end point for pedicle screw insertion using the Weinstein method. PA (pedicle angle) was the angle between lines A and B. g. Pedicle diameter (PD) was taken as the line perpendicular to line $B$, which was also taken as pedicle axis distance (PAD)

parameter regarding the distance from the lamina to the anterior cortex along the longitudinal pedicle axis was defined as pedicle axis distance (PAD), which was also used for measuring the pedicle angle (PA), as adopted by Zindrick [15] and Olsewski et al. [6] (Fig. 2) The PAD was assumed as the pedicle screw length in the Roy-Camille approach.

The anterior border of the vertebrae along the midline axis was selected as the assumed target point for pedicle screw insertion in the Weinstein approach. The distance from this assumed target point to the junction between transverse process and pedicle, which was the entry point for the Weinstein method, was measured. The distance was defined as transverse pedicle axis distance (TPAD) (Fig. 2), which was taken as the pedicle screw length in the Weinstein method.

The pedicle angle (PA) $[10,15]$, defined as the angle between the AP midline axis and pedicle axis, was measured. Moreover, the transverse intertangential angle (TITA) was defined as the angle between two lateral tangential lines along the lateral borders of each vertebra from posterior to anterior, as adopted by Van Schaik et al. [8] (Fig. 2). A positive value indicates convergence while a negative value implies divergence.

The two sides of the PA, PD, midline and pedicle axis in one vertebra were taken as independent sets of data. However, each vertebral body only had its own identical transverse intertangential angle (TITA). The mean measurements of PA, MAD, PAD and transverse pedicle diameter from L1 to L5 levels were compared with those reported by Zindrick et al. [15] Results of TITA from L3 to L5 levels obtained in this study were compared with those reported by Van Schaik et al. [8]

A total of 2905 pedicle measurements including transverse PA, MAD, PAD and transverse pedicle isthmus widths, as adopted from Zindrick et al. [15], were made from T1 to L5 using spinal CT scans and individual roentgenograms without detailed patient information. Table 1 displays individual sample sizes in each radiographic parameter. The morphology of TITA, as adopted from van Schaik et al. [8] from L3 to L5 levels was studied in 71 patients with mean age of $41.4 \pm 12.1$ years (Table 2).

Statistical analysis was performed with the SPSS for Windows statistical package, version 15.0 (SPSS, Chicago, Illinois). Independent-samples $t$ test was employed to compare mean data between Taiwanese and Caucasian subjects. A $P<0.05$ was considered statistically significant (The significance level was set at $P=0.05$ ). G*Power software (Heinrich-Heine Universit"at D"usseldorf, D"usseldorf, Germany) was utilized to calculate the power in each comparison between two groups.

\section{Results}

According to the inclusion criteria, 78 patients were selected for L1, 81 for L2, 77 for L3, 78 for L4 and 73 for L5 measurements. Significant differences $(\mathrm{P}<0.05)$ in $\mathrm{PA}$, $\mathrm{PD}, \mathrm{MAD}$ and PAD at L1 to L5 levels were observed between Caucasian and Taiwanese populations (Table 1). Moreover, significant difference $(\mathrm{P}<0.05)$ in TITA from L3 to L5 levels was also found between Caucasian and Taiwanese populations (Table 2). Van Schaik et al. [8] reported only results at L3 to L5 levels. All results obtained in this study were presented in detail in Tables 1 and 2 .

As seen in Table 1, the mean PA from L1 to L5 were $9.3^{0} \pm 1.6^{0}, \quad 7.4^{0} \pm 2.1^{0}, \quad 7.9^{0} \pm 1.8^{0}, \quad 11.2^{0} \pm 3.2^{0}, \quad 17.0^{0} \pm$ $3.1^{\circ}$, respectively; with the largest PA found at L5 level (range, $\left.9^{0}-25^{\circ}\right)$. As shown in Table 2, the mean TITA from L1 to L5 were $-12.6^{\circ} \pm 8.9^{0},-16.4^{0} \pm 8.7^{0},-10.1^{0}$ $\pm 8.1^{\circ}, 1.0^{\circ} \pm 10.3^{\circ}$ and $43.7^{\circ} \pm 14.3^{\circ}$, respectively; revealing the lower the vertebra, the greater the convergent angle along the pedicle lateral border from L3 to L5.

The mean PAD from L1 to L5 were $44.8 \pm 4.8 \mathrm{~mm}, 46.4$ $\pm 5.8 \mathrm{~mm}, 43.5 \pm 5.0 \mathrm{~mm}, 43.7 \pm 5.0 \mathrm{~mm}$ and $43.3 \pm 4.5$ 


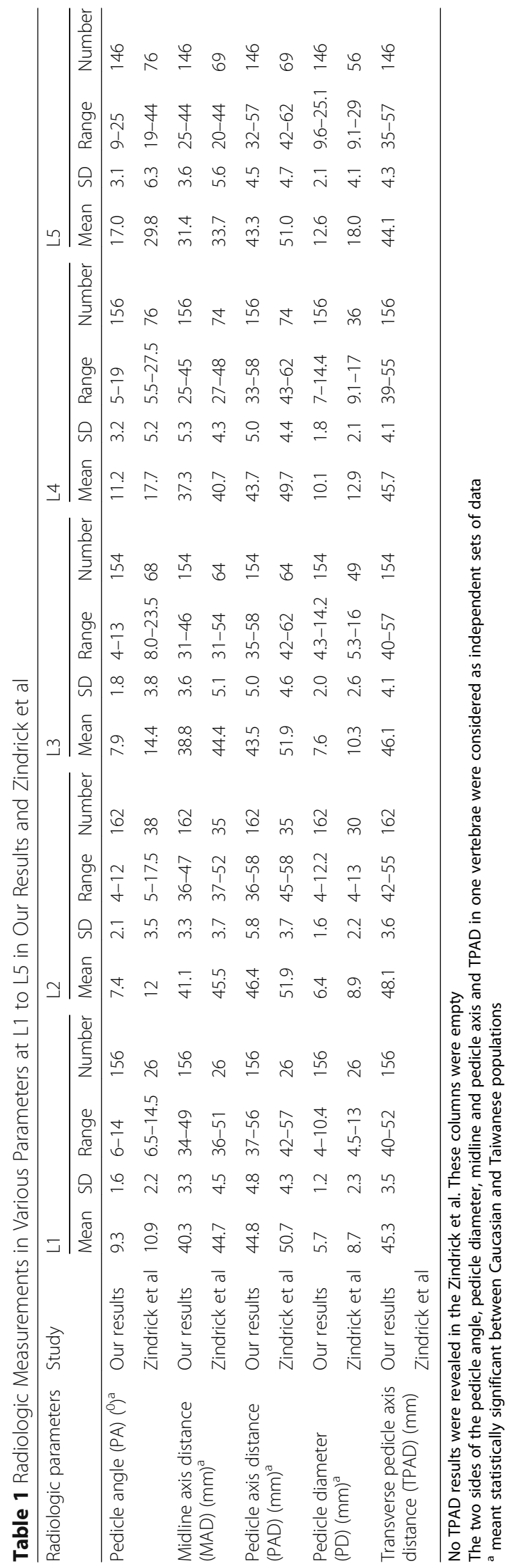


Table 2 Transverse Intertangential Angle (TITA) Between Our Results and Van Schaik et al.

\begin{tabular}{|c|c|c|c|c|c|c|c|c|c|c|c|c|c|c|c|}
\hline & \multicolumn{3}{|l|}{ L1 } & \multicolumn{3}{|l|}{ L2 } & \multicolumn{3}{|l|}{$\mathrm{L} 3{ }^{*}$} & \multicolumn{3}{|l|}{$L 4^{*}$} & \multicolumn{3}{|l|}{$L 5^{*}$} \\
\hline & Mean & SD & Range & Mean & SD & Range & Mean & SD & Range & $\overline{M e a n}$ & SD & Range & $\overline{M e a n}$ & SD & Range \\
\hline Our results & -13.4 & 7.6 & -23 to +1 & -16 & 8.3 & -29 to -5 & -9.7 & 7.5 & +5 to +29 & 2.4 & 8.5 & +4 to +21 & 44.8 & 13.1 & +16 to +78 \\
\hline Van Schaik et al & & & & & & & -14.3 & 12 & -44 to +12 & 6.3 & 11.5 & -24 to +37 & 53 & 15.4 & +18 to +88 \\
\hline
\end{tabular}

TITA is the angle between two lateral tangential lines along the lateral borders of each vertebrae was also measured from posterior-anterior axis

Positive value defined as convergence, and negative value defined as divergence from posterior-to-anterior axis

Van Schaik et al. revealed his results in $L 3$ to $L 5$ only without $L 1$ and $L 2$ results provided

* meant statistically significant between Caucasian and Taiwanese populations

$\mathrm{mm}$, respectively; indicating the more caudal the lumbar vertebra, the shorter the PAD.

The mean MAD from L1 to L5 were $40.3 \pm 3.3 \mathrm{~mm}$, $41.1 \pm 3.3 \mathrm{~mm}, 38.8 \pm 3.6 \mathrm{~mm}, 37.3 \pm 5.3 \mathrm{~mm}$ and 31.4 $\pm 3.6 \mathrm{~mm}$, respectively; with L2 having the longest MAD (range, 36-47 $\mathrm{mm}$ ) and L5 having the shortest (range, 25-44 mm).

The mean TPAD from L1 to L5 were $45.3 \pm 3.5 \mathrm{~mm}$, $48.1 \pm 3.6 \mathrm{~mm}, 46.1 \pm 4.1 \mathrm{~mm}, 45.7 \pm 4.1 \mathrm{~mm}$ and $44.1 \pm 4.3$ $\mathrm{mm}$, respectively; with $\mathrm{L} 2$ having the longest TPAD (range, 42-55), and L5 having the shortest (range, 35-57 mm).

The present results showed that L5 had the most convergent pedicle axis and shortest body distance in the anterior-posterior (AP) axis and the widest transverse pedicle diameter. L2 had the largest MAD, PAD and TPAD. The reason to explain the result is that L1 is the transitional vertebra at the thoracolumbar junction, which may have some intermediate anatomic characteristics and features. Moreover, the lower caudal lumbar vertebra had more convergent angle of PA and TITA and wider PD from L3 to L5.

Regarding power analysis, G*Power reached 0.99 for the parameters of PA, MAD, PAD and PD at L1 to L5. G*Power exceeded 0.8 for TITA at L4 and L5 (power = 0.84 and 0.85 , respectively). However, G*Power for TITA at L3 (power $=0.7$ ) was insufficient to reach a conclusion Based on the G*power software, the parameters were set up as follows: $\alpha=0.05$, equal sample sizes $(\mathrm{N} 2 / \mathrm{N} 1=1)$, power $=0.8$ and two tails in the formula. With the given results between Taiwanese (mean:-10.1, SD: 8.1) and Caucasian populations (mean:-14.3, SD:12), the recommended sample size for TITA at L3 was 95.

\section{Discussion}

This study observed difference in lumbar vertebrae morphology including PA, (PD, MAD and PAD at L1 to L5 levels between Caucasian and Taiwanese subjects. Moreover, racial differences were also found in TITA from L3 to L5 levels between Caucasian and Taiwanese populations. Only raw data after direct measurement from CT scan without age normal distribution were shown, and the number was arbitrarily chosen without prior power analysis.
There are some major weaknesses or drawbacks in this study. First, the patients did not select at the normal age distribution, which may not substantially reflect the anatomic distribution due to selection bias. Second, patients' demographic data regarding height and body weight, which may also play roles in anatomic variation, were not included in the analysis. Third, the data from both pedicles in the same vertebrae were taken as independent. Given normal symmetry, this assumption may be incorrect and thus the importance of the differences may be overestimated. Fourth, intra- and inter-observer reliability were not examined, neglecting approximately $5^{0}$ to $7^{0}$ in inter-observer variability [16]. Moreover, some assumptions may not be right, such as the PAD taken as pedicle screw length and PA taken as the trajectory angle in the Roy-Camille method. Moreover, the enrolled patients were too few to reach conclusions with adequate prior power analysis. Finally, performing post-hoc power analysis to show the adequate sample sizes was also a limitation of the study.

Pedicle angle (PA) from L1 to L5 was significantly different from those reported by Zindrick et al. [15]. In other Caucasian studies reported by Olsewski et al. [6] and Marchesi et al. [17], racial difference in PA from L1 to L5 levels as measured on radiographs was also found regardless of gender. Similar trends were observed in Caucasian and Taiwanese populations that the more caudal vertebra had larger PA with L5 having the largest. PA could be taken as the trajectory angle for pedicle screw placement in the Roy-Camille method. Apparently, surgeons performing the Weinstein approach with free-hand technique would prefer more convergent angle with larger PA. In pedicle screw placement, surgeons had to keep pedicle screws within the pedicle to achieve maximum fixation and pullout-resisting strength and avoid iatrogenic complications regardless of the method adopted.

Regarding TITA, the more caudal vertebra had more convergent pedicle axis with L5 having the largest TITA, as seen in the results of this study and Zindrick et al. [8] L5 had the largest PA and TITA but shortest MAD, PAD and TPAD. Consequently, L5 vertebra has more hemispherical morphology with more convergent pedicle axis 
from the posterior-anterior axis $[18,19]$ in relation to the L5 vertebral body. Hence, it is easy to break through the anterior cortex when adopting the Roy-Camille method for $\mathrm{L} 5$ pedicle screw placement due to the shorter AP axis and greater convergence along the pedicle axis. Degenerative spondylolisthesis occurs most commonly at the L4-L5 level [20], and surgeons have to keep in mind the unique morphology of L5 when placing pedicle screws with either method via free-hand technique.

Regarding pedicle axis distance (PAD) and midline axis distance (MAD), the present study found that L2 had the largest PAD and MAD, which is consistent with the finding of Zindrick et al. [15]. Among the Caucasian population, Olsewski et al. [6] reported that L4 had the largest posterior-to-anterior distance. Either PAD or MAD may be taken as a reference for the pedicle screw length when performing the Roy-Camille method according to the surgeons' preferences with different trajectory angles. Increased screw length could enhance fixation strength of the pedicle screw within the bone [21]. However, iatrogenic complication could occur if the screws penetrate into the abdominal cavity.

No matter which method was applied for pedicle screw placement, surgeons should carefully use the guide pin and pedicle finder to check the tract length and its convergence with repeated checks using portable $\mathrm{C}$-arm images. Proper length preparation in the vertebral body could avoid anterior penetration into the abdominal cavity so as to avoid complications including vascular injury and hallow organ perforation. Hirano et al. [22] pointed out that the pedicle of spine plays a more important role in resisting pullout strength than the vertebrae. $\mathrm{Li}$ et al. [23] reported an approximate $23.4 \%$ decrease in pullout strength of pedicle screws under lateral wall perforation. Therefore, surgeons had to make sure that the screw is within the pedicle without medial or anterior or lateral wall perforation when performing either method although some surgeons may adopt the "in-out-in" technique for pedicle screw placement.

Pedicle diameter (PD) may be used as a reference for surgeons when choosing the pedicle screw diameter. This study and Zindrick et al. [15] found that L5 had the largest $\mathrm{PD}$, and the more caudal lumbar vertebra had larger PD. Similar results were also observed among Caucasians as reported by Olsewski et al. [6] Wittenberg et al. [24] reported that pedicle screw of larger diameter increased axial pullout force and enhanced spinal stability, but might have higher incidence of breaking through the pedicle medial wall and further damage nerve root, especially with inappropriate convergent angle. Misenhimer et al. [25] indicated that the ideal screw diameter was $80 \%$ of PD. According to the present results, the ideal pedicle screw diameter from L1 to L5 were $4.7 \mathrm{~mm}, 5.9 \mathrm{~mm}, 6$ $\mathrm{mm}, 8.1 \mathrm{~mm}$ and $12.1 \mathrm{~mm}$, respectively. Hence, the diameter of $6.0 \mathrm{~mm}$ could be safely used in the Taiwanese population with both methods for lower lumbar pedicle screw fixation (L3 to L5).

Owing to individualized variability among patients and populations, surgeons need to be aware of the possible lumbar morphology and pedicle orientation when placing pedicle screws with either the Roy-Camille or Weinstein method using free-hand technique or guided by navigation system. Meticulous pre-operative planning with CT scan is important to ensure appropriate diameter, length and trajectory for pedicle screw placement.

The results obtained illustrated variability of each lumbar morphology and pedicle orientation and differences between Caucasian and Taiwanese populations. Such information may help surgeons place the lumbar pedicle screws more safely and accurately to avoid iatrogenic complications. Moreover, meticulous pre-operative planning and intra-operative multiple portable $\mathrm{C}$-arm checkup, guide pin with pedicle finder systems could also be helpful to alleviate iatrogenic complications if the $\mathrm{O}$-arm navigation system is not routinely used in clinics.

\section{Abbreviations}

MAD: Midline axis distance; PA: Pedicle angle; PAD: Pedicle axis distances; PD: Pedicle diameter; TITA: Transverse intertangential angle; TPAD: Transverse pedicle axis distance

\section{Acknowledgements \\ We thank Hsin-Yi Huang from the Biostatistics Task Force, Taipei Veterans General Hospital, for assistance in statistical analysis.}

\section{Funding}

No funding for the research reported needed to be declared.

\section{Availability of data and materials}

All data generated or analyzed during this study are included in this published article.

\section{Authors' contributions}

HHL: acquisition of data, writing manuscript, JPW: analysis and interpretation of data. CLL: analysis and interpretation of data. YCY: analysis and interpretation of data, STW: analysis and interpretation of data, writing manuscript, MCC: analysis and interpretation of data. PHC: conception and design, drafting manuscript. All authors read and approved the final manuscript.

\section{Ethics approval and consent to participate}

The research protocol was approved by the institutional review board of Taipei Veterans General Hospital. Verbal consent from patients was taken over the phone.

\section{Consent for publication \\ Not applicable.}

\section{Competing interests}

The authors declare that they have no competing interests. No benefits in any form have been received or will be received from a commercial party related directly or indirectly to the subject of this article.

\section{Publisher's Note}

Springer Nature remains neutral with regard to jurisdictional claims in published maps and institutional affiliations. 


\section{Author details}

'Department of Orthopedics \& Traumatology, Taipei Veterans General Hospital, No. 201, Sec. 2, Shipai Rd., Beitou District, Taipei City 11217, Taiwan, Republic of China. ${ }^{2}$ School of Medicine, National Yang-Ming University, No. 201, Sec. 2, Shipai Rd., Beitou District, Taipei City 11217, Taiwan, Republic of China. ${ }^{3}$ Department of Orthopaedic Surgery, National Cheng Kung University Hospital, College of Medicine, National Cheng Kung University, Tainan, Taiwan, Republic of China.

Received: 23 October 2017 Accepted: 30 April 2019

Published online: 25 May 2019

\section{References}

1. Genant HK, Wu CY, van Kuijk C, Nevitt MC. Vertebral fracture assessment using a semiquantitative technique. J Bone Miner Res Off J Am Soc Bone Miner Res. 1993;8(9):1137-48.

2. Gelalis ID, Paschos NK, Pakos EE, Politis AN, Arnaoutoglou CM, Karageorgos AC, Ploumis A, Xenakis TA. Accuracy of pedicle screw placement: a systematic review of prospective in vivo studies comparing free hand, fluoroscopy guidance and navigation techniques. European Spine Journal: official publication of the European Spine Society, the European Spinal Deformity Society, and the European Section of the. Cervical Spine Research Society. 2012;21(2):247-55.

3. Roy-Camille R, Saillant G, Mazel C. Internal fixation of the lumbar spine with pedicle screw plating. Clin Orthop Relat Res. 1986;(203):7-17.

4. Weinstein JN, Spratt KF, Spengler D, Brick C, Reid S. Spinal pedicle fixation: reliability and validity of roentgenogram-based assessment and surgical factors on successful screw placement. Spine. 1988;13(9):1012-8.

5. Holly LT, Schwender JD, Rouben DP, Foley KT. Minimally invasive transforaminal lumbar interbody fusion: indications, technique, and complications. Neurosurg Focus. 2006;20(3):E6.

6. Olsewski JM, Simmons EH, Kallen FC, Mendel FC, Severin CM, Berens DL. Morphometry of the lumbar spine: anatomical perspectives related to transpedicular fixation. The Journal of bone and joint surgery American. 1990;72(4):541-9.

7. Vaccaro AR, Rizzolo SJ, Allardyce TJ, Ramsey M, Salvo J, Balderston RA, Cotler $J \mathrm{M}$. Placement of pedicle screws in the thoracic spine. Part I: morphometric analysis of the thoracic vertebrae. J Bone Joint Surg Am. 1995;77(8):1193-9.

8. van Schaik JJ, Verbiest H, van Schaik FD. Morphometry of lower lumbar vertebrae as seen on CT scans: newly recognized characteristics. AJR Am J Roentgenol. 1985;145(2):327-35.

9. Mitra SR, Datir SP, Jadhav SO. Morphometric study of the lumbar pedicle in the Indian population as related to pedicular screw fixation. Spine. 2002;27(5):453-9.

10. Chadha M, Balain B, Maini L, Dhaon BK. Pedicle morphology of the lower thoracic, lumbar, and S1 vertebrae: an Indian perspective. Spine. 2003;28(8):744-9.

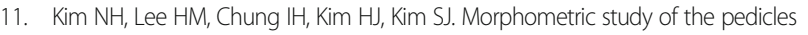
of thoracic and lumbar vertebrae in Koreans. Spine. 1994;19(12):1390-4.

12. Hou S, Hu R, Shi Y. Pedicle morphology of the lower thoracic and lumbar spine in a Chinese population. Spine. 1993;18(13):1850-5.

13. Chou PH, Shyu JF, Ma HL, Wang ST, Chen TH. Courses of the radial nerve differ between Chinese and Caucasians: clinical applications. Clin Orthop Relat Res. 2008;466(1):135-8.

14. Beers GJ, Carter AP, Leiter BE, Tilak SP, Shah RR. Interobserver discrepancies in distance measurements from lumbar spine CT scans. Am J Roentgenol. 1985;144(2):395-8.

15. Zindrick MR, Wiltse LL, Doornik A, Widell EH, Knight GW, Patwardhan AG, Thomas JC, Rothman SL, Fields BT. Analysis of the morphometric characteristics of the thoracic and lumbar pedicles. Spine. 1987;12(2):160-6.

16. Morrissy RT, Goldsmith GS, Hall EC, Kehl D, Cowie GH. Measurement of the cobb angle on radiographs of patients who have scoliosis. Evaluation of intrinsic error. J Bone Joint Surg Am. 1990;72(3):320-7.

17. Marchesi D, Schneider E, Glauser P, Aebi M. Morphometric analysis of the thoracolumbar and lumbar pedicles, anatomo-radiologic study. Surgical andRadiologic anatomy. 1988;10(4):317-22.

18. Wang S, Wang Q, Kang J, Xiu P, Wang G. An imaging anatomical study on percutaneous kyphoplasty for lumbar via a unilateral transverse processpedicle approach. Spine. 2014;39(9):701-6.

19. Alfonso M, Palacio P, Bastarrika G, Villas C. Does the shape of the $L 5$ vertebral body depend on the height of $C T$ slices in the pedicle? Evaluation of the shape of the L5 vertebral body with a multicut CT scan. Spine. 2008;33(1):E1-5.
20. Jacobsen S, Sonne-Holm S, Rovsing H, Monrad H, Gebuhr P. Degenerative lumbar spondylolisthesis: an epidemiological perspective: the Copenhagen osteoarthritis study. Spine. 2007;32(1):120-5.

21. Zindrick MR, Wiltse LL, Widell EH, Thomas JC, Holland WR, Field BT, Spencer CW. A biomechanical study of intrapeduncular screw fixation in the lumbosacral spine. Clin Orthop Relat Res. 1986;(203):99-112.

22. Hirano T, Hasegawa K, Takahashi HE, Uchiyama S, Hara T, Washio T, Sugiura T, Yokaichiya M, lkeda M. Structural characteristics of the pedicle and its role in screw stability. Spine. 1997;22(21):2504-9 discussion 2510. (???

23. Li N, He D, Xing $Y, L v Y$, Tian $W$. The effect of lateral wall perforation on screw pull-out strength: a cadaveric study. J Orthop Surg Res. 2015;10:6.

24. Wittenberg RH, Lee KS, Shea M, White AA 3rd, Hayes WC. Effect of screw diameter, insertion technique, and bone cement augmentation of pedicular screw fixation strength. Clin Orthop Relat Res. 1993;(296):278-287.

25. Misenhimer GR, Peek RD, Wiltse LL, Rothman SL, Widell EH Jr. Anatomic analysis of pedicle cortical and cancellous diameter as related to screw size. Spine. 1989;14(4):367-72.

\section{Ready to submit your research? Choose BMC and benefit from:}

- fast, convenient online submission

- thorough peer review by experienced researchers in your field

- rapid publication on acceptance

- support for research data, including large and complex data types

- gold Open Access which fosters wider collaboration and increased citations

- maximum visibility for your research: over $100 \mathrm{M}$ website views per year

At BMC, research is always in progress.

Learn more biomedcentral.com/submissions 